\title{
Nachträge zu den Tafeln zur Reduction von Sterncatalogen auf das System des Fundamentalcatalogs der A.G. und zu den Gewichtstafeln für Sterncataloge.
}

\author{
Von A. Auwers.
}

Bei der Zusammenstellung der Tafeln zur Reduction auf das System des Fundamentalcatalogs für die Zonenbeobachtungen der Astronomischen Gesellschaft (A. N. 3195-6, 3413-4 und 3463) habe ich eine Anzahl von Tafeln bei Seite gelassen, deren Mittheilung mir aus dem Grunde nach der Einverleibung der betr. Cataloge in den neuen Fundamentalcatalog kein weiteres Interesse zu haben schien, weil diese Cataloge nur Objecte des letztern oder fast nur solche enthalten. Es ist jedoch wünschenswerth die Grundlagen für die systematische Correctur des Fundamentalcatalogs von 1879 - die derart vorzunehmen ist, daß das System des neuen Catalogs zwischen den Systemen aller stimmfähigen Instrumente die Mitte hält - vollständig nachzuweisen, und es ist auch abgesehen von allen Anwendungen nicht ohne Interesse, das Verhalten der in den früheren Mittheilungen übergangenen Cataloge, unter denen sich einige für die Bearbeitung des Fundamentalcatalogs besonders wichtige befinden, ebenfalls näher festzustellen.

Es sind ferner seit meiner letzten Mittheilung über den Gegenstand (1897) einige Cataloge theils erschienen theils durch gefälligst von den Bearbeitern flir mich angefertigte Auszüge verfügbar geworden, für welche ich ebenfalls Reductionstafeln aufgestellt habe.

Diese beiden Gruppen von Tafeln sind auf den folgenden Seiten, in jeder Beziehung sich an die früheren Mit. theilungen anschließend, zusammengestellt.

Unter der Ueberschrift $\gtrsim$ Zweiter Theil der Reduction* findet man häufig vo\& angegeben oder eine Reihe von Werthen, die ebenso thatsächlich nur nachweisen sollen, daß dieser zweite Theil durchweg unmerklich ist. Ich habe die zu verschiedenen Zeiten abgeleiteten einzelnen Tafeln unverändert so gegeben, wie ich sie in meinen Rechnungen aufgestellt und benutzt habe. Die Angabe $>$ \& zeigt an, daß sich als Stundenmittel der nach Anbringung des »ersten Theils der Reduction « übrig bleibenden Reste der Abweichungen vom A.G.C. lediglich als zufällige Beobachtungsfehler anzusehende Werthe gefunden haben und alsdann vernachlässigt worden sind; in einzelnen Fällen ist gleichwohl noch die gewöhnliche Ausgleichung vorgenommen und ihr Ergebnil hier mitgetheilt. Wo die Columnen für den zweiten Theil der Reduction einfach leer gelassen sind, bedeutet dieß, daß bei streng differentiellem Aaschluß der Beobachtungsreihe an den A.G.C. die Entstehung von Unterschieden die mit der RA. fortschreiten von vorn herein als ganz ausgeschlossen angesehen worden ist.
Im einzelnen ist $z u$ den Reductionstafeln noch fol. gendes zu bemerken.

Groombridge, neue Bearbeitung von Thackeray. Der mir von Mr. Thackeray zuerst mitgetheilte und hier zu Grunde gelegte Auszug gibt die Resultate der Beobachtungen der Jahre $1806-1810$, von denen die inzwischen mit $\mathrm{Zu}$ ziehung der weiteren Jahre 18 1 I-1 8 I 6 endgültig festgestellten Oerter sich systematisch nicht unterscheiden.

Königsberg I8I5. - Die Tafeln beziehen sich auf die von Dr. Cohn ausgeführte Bearbeitung der Beobachtungen am Doilond'schen Passageninstrument und am Caryschen Kreis. Die noch nicht veröffentlichten Declinationen wurden mir von Hrn. Cohn mitgetheilt, außerdem das Resul. tat einer Vergleichung mit Argelander's Aboer Catalog. Die Reductionstafeln sind auf letztere gegründet, da sie so durch erheblich größere Anzahl der gemeinschaftlichen Objecte besser gesichert wurden als durch directe Vergleichung Kön.-A.G.C. Für den Himmelstheil zwischen $+45^{\circ}$ und dem Pol gilt $\Delta \delta_{\delta}$ für Mittel aus oberen und unteren Culminationen.

Pond 1815: meine Bearbeitung der Beobachtungen am Troughton'schen Passageninstrument i 8 I6-i8 ig und am Mauerkreis 1812-1819, Abh. d. Berl. Akad. r9o1. Der an diesem Orte, Math. Abh.I S. 266-30r, gegebene Catalog von 570 Sternen tritt an die Stelle der sämmtlichen Pond'schen Cataloge für 1813 , I815 und 1817 .

Taylor 1835 , neue Ausgabe von Downing. - In $5^{\circ}$ breiten Zonen (in der südlichsten zwischen $-25^{\circ}$ und $-31^{\circ}$ ) finden sich nach Anbringung der Reduction $\Delta \delta_{\alpha}$ folgende Mittelwerthe der Unterschiede T. - A.G.C.:

$$
\begin{aligned}
& 77^{\circ} .7+0.03 \quad 17 \text { St. } 22 \% 6+0.87 \quad 31 \text { St. } \\
& 72.2-0.22 \quad 19 » \quad 17.5+0.6833 \\
& 67.2-1.0327 \% \quad 12.7+1.0430 \\
& 62.0-0.8022 》 7.8+0.5731 \text { \% } \\
& 57.6-0.5531 \% 2.8+I .1528 》 \\
& 5^{2.3}-0.6720>-2.6+0.7525 \text {. } \\
& 47.4-0.6 \text { I } 29 *-7.9+0.7821 \% \\
& 42.4-0.3^{1} 32 *-12.6+1.0222 \\
& 38.0+0.1136 *-16.7+0.8525 \text { » } \\
& 32.6+0.1627 \text {-22.4 +0.66 } 20 \text { 》 } \\
& 27.8-0.0633 *-28.5+1.5313 \text { }
\end{aligned}
$$

Die Annahme einer durch die Taylor'sche Theilungsfehlertafel nicht ausgeglichenen Discontinuität bei $+25^{\circ}$ erscheint nothwendig. 
Edinburg 1840. - Die Bearbeitung des Catalogs, den Dr. Halm aus den Beobachtungen der Jahre $1836-1845$ herstellt, ist noch nicht ganz abgeschlossen. Ich habe die von Hrn. Halm für mich ausgezogenen Jahresresultate benutzt; dabei fand sich für die Rectascensionen eine Trennung der beiden Gruppen 1836-1839 und 1840-1845 nothwendig, die sich sowohl hinsichtlich ihres Verhaltens zum A.G.C. als hinsichtlich der Genauigkeit der Resultate unterscheiden.

Sydney 1860: die beiden Jahrgänge der Scott'schen Beobachtungen 1859 und 1860. - Die Tafel für $4 \alpha_{c \ell}$ gibt die Reduction des zu Grunde gelegten Fundamentalcatalogs (N. A. 1860).

Madras 1875, Generalcatalog der 1862-1887 beobachteten Sterne. - Die Declinationen müssen zunächst noch von einem bei der Vergleichung seht deutlich hervortretenden Theilungsfehlergliede $-0.65 \cos 12\left(\delta+5^{\circ}\right)$ befreit werden, durch Anbringung folgender Correctionen:

$\begin{array}{llllll}-30.0 & 0.0 & 30 & 0 & 60 & 0 \\ -29 & 1 & 31 & 61 & +0.32 \\ -28 & 2 & 32 & 62 & +0.20 \\ -27 & 3 & 33 & 63 & -0.07 \\ -26 & 4 & 34 & 64 & -0.20 \\ -25 & 5 & 35 & 65 & -0.32 \\ -24 & 6 & 36 & 66 & -0.44 \\ -23 & 7 & 37 & 67 & -0.53 \\ -22 & 8 & 38 & 68 & -0.60 \\ -21 & 9 & 39 & 69 & -0.63 \\ -20 & 10 & 40 & 70 & -0.65 \\ -19 & 11 & 41 & 71 & -0.63 \\ -18 & 12 & 42 & 72 & -0.60 \\ -17 & 13 & 43 & 73 & -0.53 \\ -16 & 14 & 44 & 74 & -0.44 \\ -15.0 & 15.0 & 45.0 & 75.0 & -0.32\end{array}$

$$
\begin{array}{llllll}
-15^{\circ} 0 & 155^{\circ} 0 & 45^{\circ} \circ & 75^{\circ} 0 & -0.32 \\
-14 & 16 & 46 & 76 & -0.20 \\
-13 & 17 & 47 & 77 & -0.07 \\
-112 & 18 & 48 & 78 & +0.07 \\
-11 & 19 & 49 & 79 & +0.20 \\
-10 & 20 & 50 & 80 & +0.32 \\
-9 & 21 & 51 & 81 & +0.44 \\
-8 & 22 & 52 & 82 & +0.53 \\
-7 & 23 & 53 & 83 & +0.60 \\
-6 & 24 & 54 & 84 & +0.63 \\
-5 & 25 & 55 & 85 & +0.65 \\
-4 & 26 & 56 & 86 & +0.63 \\
-3 & 27 & 57 & 87 & +0.60 \\
-2 & 28 & 58 & 88 & +0.53 \\
-1 & 29 & 59 & 89 & +0.44 \\
0.0 & 30.0 & 60.0 & 90.0 & +0.32
\end{array}
$$

Für die so verbesserten Declinationen gilt die unten für $4 \delta_{\delta}$ gegebene Tafel.

Sydney 1878 : Die beiden Russell'schen Jahrgänge 1877 und 1878 .

Pulkowa 1885 , P.I. und V.Kr. - In RA. findet sich eine sehr auffällige Abweichung in den Stunden 18-20: während die $26 \mathrm{I}$ südlich vom Zenith culminirenden Sterne der übrigen 2 I Stunden Pu. + $\Delta \alpha_{\delta}-$ A.G.C. im Mittel genau $=0$ geben, findet sich für diese 3 Stunden aus 42 Sternen das Mittel +0.or 7 , oder nach Anbringung der vorläufigen Correctionen an die Rectascensionen des A.G.C. (A.N.3508-9) + $0^{\mathrm{s}} .15$. Zwischen $18^{\mathrm{h}} \mathrm{I} 5^{\mathrm{m}}$ und $20^{\mathrm{h}} 45^{\mathrm{m}}$ geben von 39 Sternen 36 den Unterschied + und nur $3-$; ich habe für diese $2 \frac{1}{2}$ Stunden - o.or 5 als Specialreduction angenommen.

Pulkowa 1885 , Mer. Kr. - Der zweite, noch nicht ausgegebene Romberg'sche Catalog, dessen Bearbeitung Hr. Seyboth zu Ende geführt hat, enthält die Sterne des A.G.C. wiederum fast vollzählig. Die von Hrn. Seyboth mir mitgetheilten Oerter derselben gaben in RA. zunächst merklich größere durchschnittliche Unterschiede als der Catalog für 1875, indem sich in den späteren Rectascensionen eine Helligkeitsgleichung stark bemerklich macht. Außer den $\Delta \alpha_{\delta}$ der unten gegebenen Reductionstafel sind deshalb noch folgende Correctionen, die ich als gültig bis $\delta==+60^{\circ}$ angesehen habe, anzubringen:

$$
\text { für Hell. } \begin{array}{cc}
0.8 & -0.024 \\
1.2 & -0.023 \\
1.6 & -0.021 \\
2.0 & -0.019 \\
2.4 & -0.016 \\
2.8 & -0.013 \\
3.2 & -0.010 \\
3.6 & -0.006 \\
4.0 & -0.001 \\
4.4 & +0.003 \\
4.8 & +0.008 \\
5.2 & +0.014 \\
5.6 & +0.020 \\
6.0 & +0.027 \\
6.4 & +0.035
\end{array}
$$

In den Berliner Rectascensionen von Romberg zeigt sich keine Spur einer Helligkeitsgleichung gegen A.G.C. Bei der Vergleichung der ersten Pulkowaer Reihe drängte sich eine solche ebenfalls nicht der Wahrnehmung auf, nachdem sie sich aber in der späteren Reihe als unzweifelhaft vorhanden gezeigt hat, darf man die in der mittleren der drei Reihen verbleibenden Unterschiede auf Anfänge einer Helligkeitsgleichung deuten. Es findet sich nämlich die nach Anbringung der übrigen Reductionen übrig bleibende Abweichung von A.G.C. für die Romberg'schen Rectascensionen: 


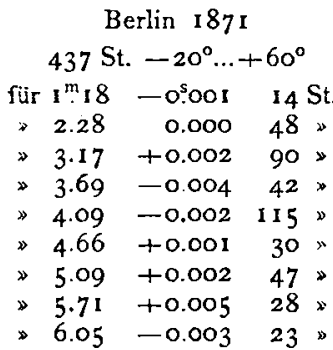

\begin{tabular}{|c|c|c|}
\hline \multicolumn{3}{|c|}{ Pulkowa 1875} \\
\hline$r 1^{m}=20$ & 0.000 & $13 \mathrm{St}$ \\
\hline 2.29 & +0.001 & $47 \gg$ \\
\hline 3.16 & +0.002 & $93 »$ \\
\hline 3.67 & +0.002 & 462 \\
\hline 4.08 & $-0.00 \mathrm{I}$ & 120 \\
\hline 4.67 & -0.002 & 28 \\
\hline 5.09 & -0.008 & $45 \gg$ \\
\hline 5.70 & 0.000 & $29 \gg$ \\
\hline 6.07 & -0.004 & 23 \\
\hline
\end{tabular}

\begin{tabular}{|c|c|c|c|}
\hline \multicolumn{2}{|c|}{366 St. $-10^{\circ} \ldots+50^{\circ}$} & & \\
\hline fiir & $I^{m} \cdot 20$ & +0.023 & 12 St. \\
\hline & 2.33 & +0.014 & 36 \\
\hline & 3.1 & +0.0II & 76 \\
\hline & 3.6 & +0.006 & 42 \\
\hline & 4.08 & -0.005 & 104 \\
\hline & 4.66 & +0.003 & 19 \\
\hline & $5.1 \mathrm{I}$ & -0.008 & 38 \\
\hline & 5.7 & -0.028 & $21 \%$ \\
\hline & 6.08 & -0.023 & $18 \%$ \\
\hline
\end{tabular}

Es scheint hier also ein Fall einer fortschreitenden Aenderung des Beobachters vorzuliegen, und zwar in entgegengesetzter Richtung zu den von Hrn. de Ball A.N. 3857 erörterten Beispielen so, daß die absolute Helligkeitsgleichung des Beobachters sich fortgesetzt verringert hat, denn die Abweichung für Pulkowa I885 wird ungefähr mit der Correction übereinstimmen, welche an die Rectascensionen des A.G.C. anzubringen ist.

Madison 1885 , Holden. - In Bezug auf die Reduction $\Delta \delta_{\delta}$ sind die Hauptreihe der Beobachtungen von Holden, Comsteck und Updegraff Mai 1884 - Oct. 1885 mit dem Nachtrag von Updegraff von Nov. und Dec. I 885, Publ. Washb. Obs. Vol. IV, und die beiden Nachträge des Vol. V zu unterscheiden, indem für letztere zu den für die Hauptreihe gefundenen $\Delta \delta_{\delta}$ noch die folgenden Reductionen hinzuzufügen waren:

Reihe $1886-87$, Vol. V Div. V

+0.55 für Südsterne und $\delta$ aus U.C.,

-0.55 für Nordsterne O.C.

Reihe April-Mai I 887, Vol. V App.

für Südsterne +0 ". 14 ,

für Nordsterne O.C. -0.54, U.C. +o. 54 .

Die Reductionstafel für die Hauptreihe ist für U.C. durch folgenden Zusatz zu ergänzen:

$\begin{array}{llr}90^{\circ} \Delta \alpha_{\delta} & \Delta \delta_{\delta}+0.07 \\ 85 & +0.052 & -0.30 \\ 80 & +0.017 & -0.65 \\ 75 & +0.008 & -0.94 \\ 70 & -0.003 & -0.77 \\ 65 & -0.014 & -0.24 \\ 60 & -0.023 & +0.25\end{array}$

Madison 1890 , Brown. - Fortsetzung der Tafel für U.C.:

$\begin{array}{lrr}90^{\circ} & \Delta \alpha_{\delta} & \Delta \delta_{\delta}-0.02 \\ 85 & -0.103 & +0.04 \\ 80 & -0.046 & +0.05 \\ 75 & -0.023 & +0.03 \\ 70 & -0.006 & 0.00 \\ 65 & +0.009 & -0.03 \\ 60 & +0.024 & -0.06\end{array}$

Melbourne 1890: Auszug aus dem MS. des Third Melbourne General Catalogue, von Mr. Baracchi mitgetheilt.

San Fernando I892-93. - Die Werthe $\Delta \alpha_{\alpha}$ für Decl. $+60^{\circ}$ bis $+85^{\circ}$ gelten für die Beobachtungen des Jahres 1892 , in welchem allein Rectascensionen von Circumpolarsternen beobachtet sind. Im übrigen geben die Tafeln ein Mittel der, wenig ubereinstimmenden, aus den beiden Jahrgängen einzeln abgeleiteten.

Ottakring 1897 , Großmann. - Die Tafel gilt für die vorläufig (Abh.d. Sächs. Ges. d.W., Bd. 27 der math. ph. Cl.) mitgetheilten Declinationen, deren definitive systematische Feststellung Dr. Großmann sich noch vorbehalten hat.

München 1900: unveröffentlichte, von Dr. Oertel zur Verfügung gestellte Bestimmungen. Die Declinationen sind von Hrn. Oertel in verschiedenen Annahmen für die Reductionselemente abgeleitet, die unten gegebenen $\Delta \delta_{\delta}$ gelten für das mit der Bauschinger'schen Refraction und der ihr entsprechenden Polhöhe aufgestellte System. Für die aus U.C. abgeleiteten Declinationen wird die Fortsetzung der Tafel:

$$
\begin{array}{ll}
85^{\circ} \Delta \delta_{\delta}= & +0.03 \\
80 & +0.21 \\
75 & +0.40
\end{array}
$$

Die Durchgänge sind mit dem Registrirmikrometer beobachtet und die Rectascensionen deshalb von einer Helligkeitsgleichung, mehr oder weniger vollständig, frei; demgemäß kommt in der Vergleichung mit A.G.C. eine wesentlich diesern eigenthümliche Helligkeitsgleichung zum Vorschein und es ist außer $\Delta \alpha_{\alpha}$ noch die folgende Reduction in Rechnung zu bringen:

$\begin{array}{cccc}\mathrm{rm}^{\mathrm{m}} \mathrm{o} & -0.023 & 4^{\mathrm{m}} \circ & +0.001 \\ 1.5 & -0.017 & 4.5 & +0.003 \\ 2.0 & -0.012 & 5.0 & +0.004 \\ 2.5 & -0.008 & 5.5 & +0.005 \\ 3.0 & -0.004 & 6.0 & +0.006 \\ 3.5 & -0.001 & 6.5 & +0.006\end{array}$

Greenwich 1890 : Second Ten-year Catalogue (Beob. 1887-1896). - Neben der für die Catalogangaben gültigen Tafel der $\Delta \delta_{\delta}$ ist noch eine zweite Col. aufgestellt, welche die Reduction für solche Declinationen angibt, wie sie bei unveränderter Beibehaltung der in Greenwich 1880-1896 benutzten Theilungsfehler erhalten worden wären. So wurden mir die Oerter 1897 von Mr. Christie mitgetheilt und schließen sich so im ganzen dem A.G.C. noch etwas enger an. 


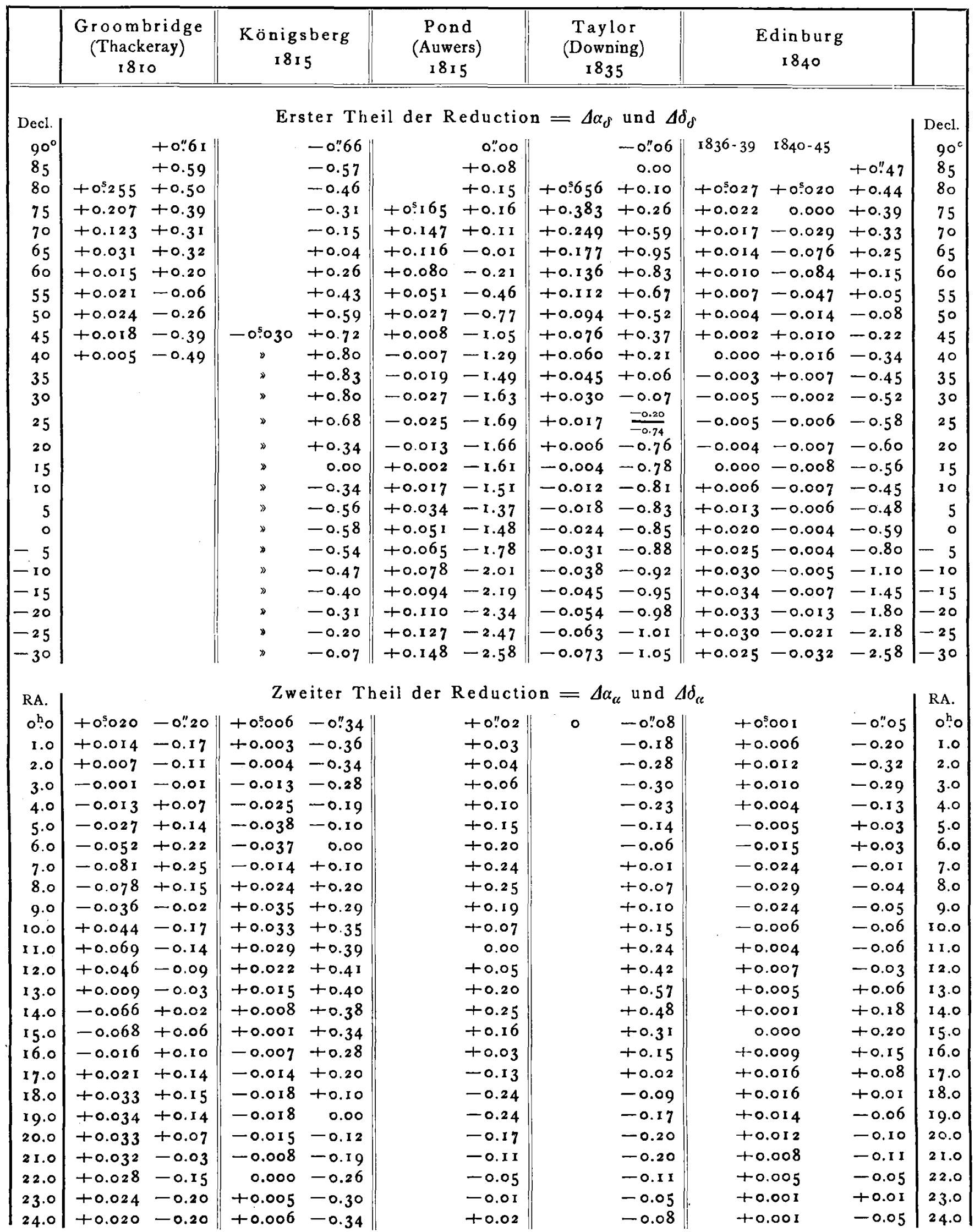




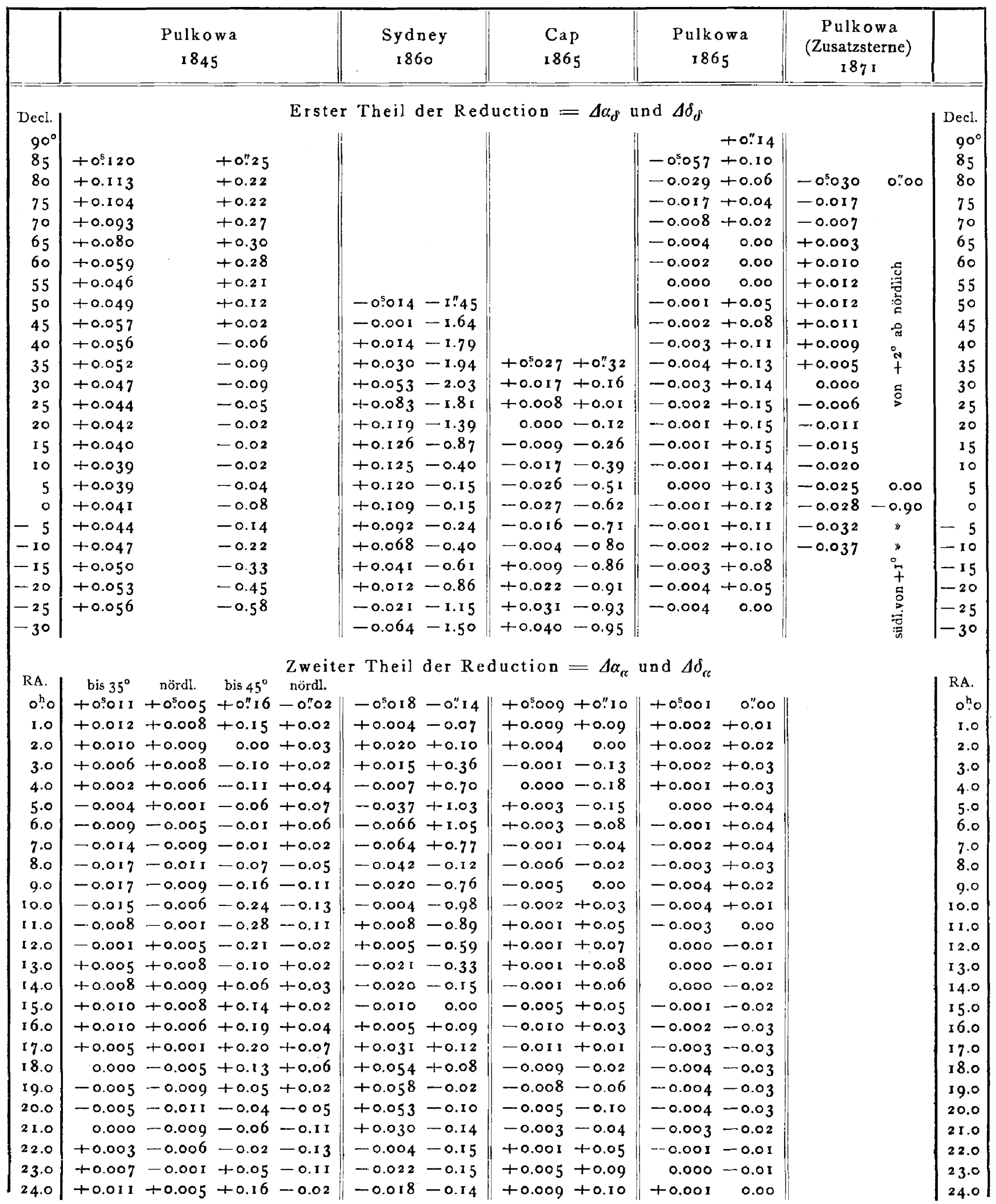




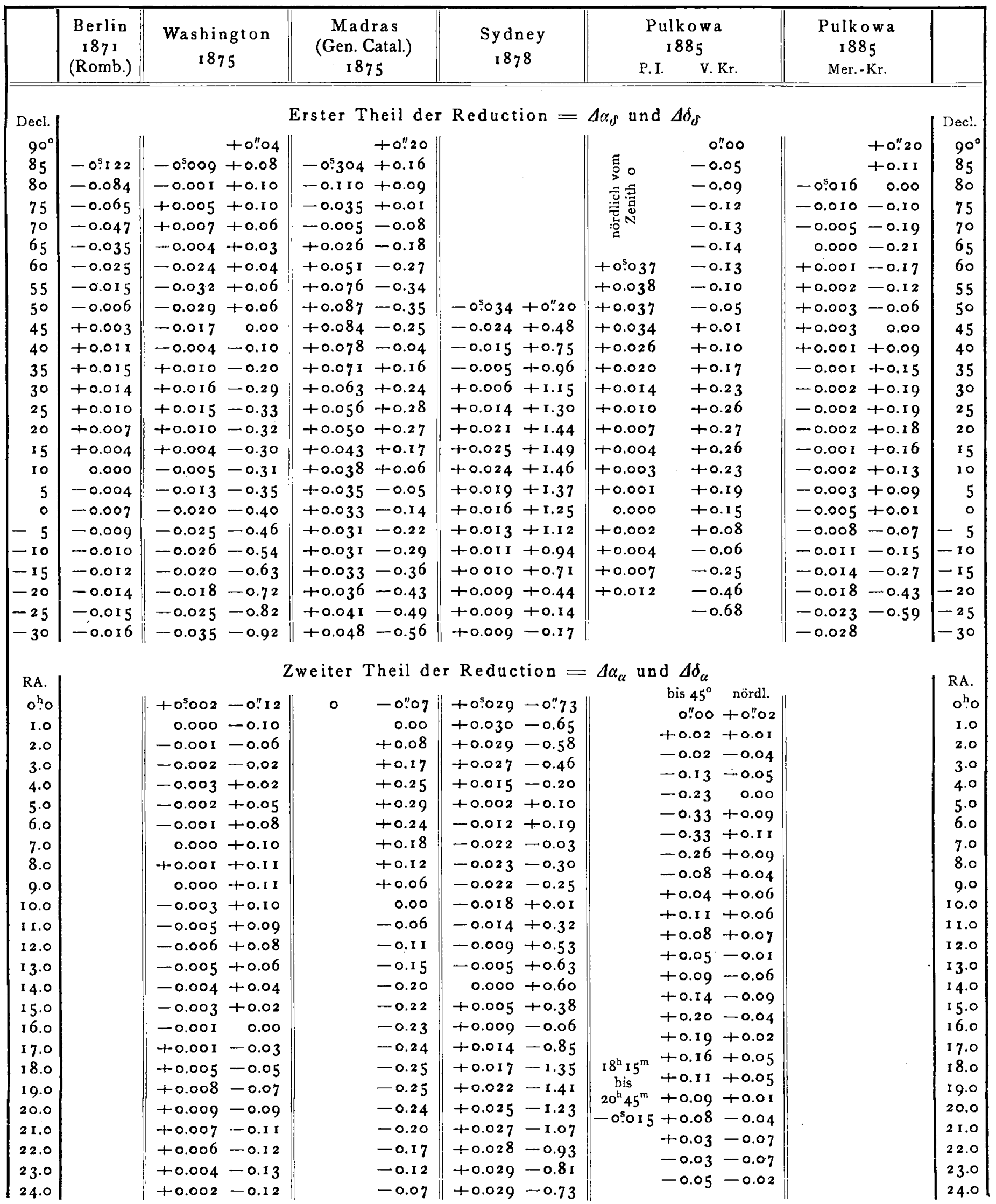




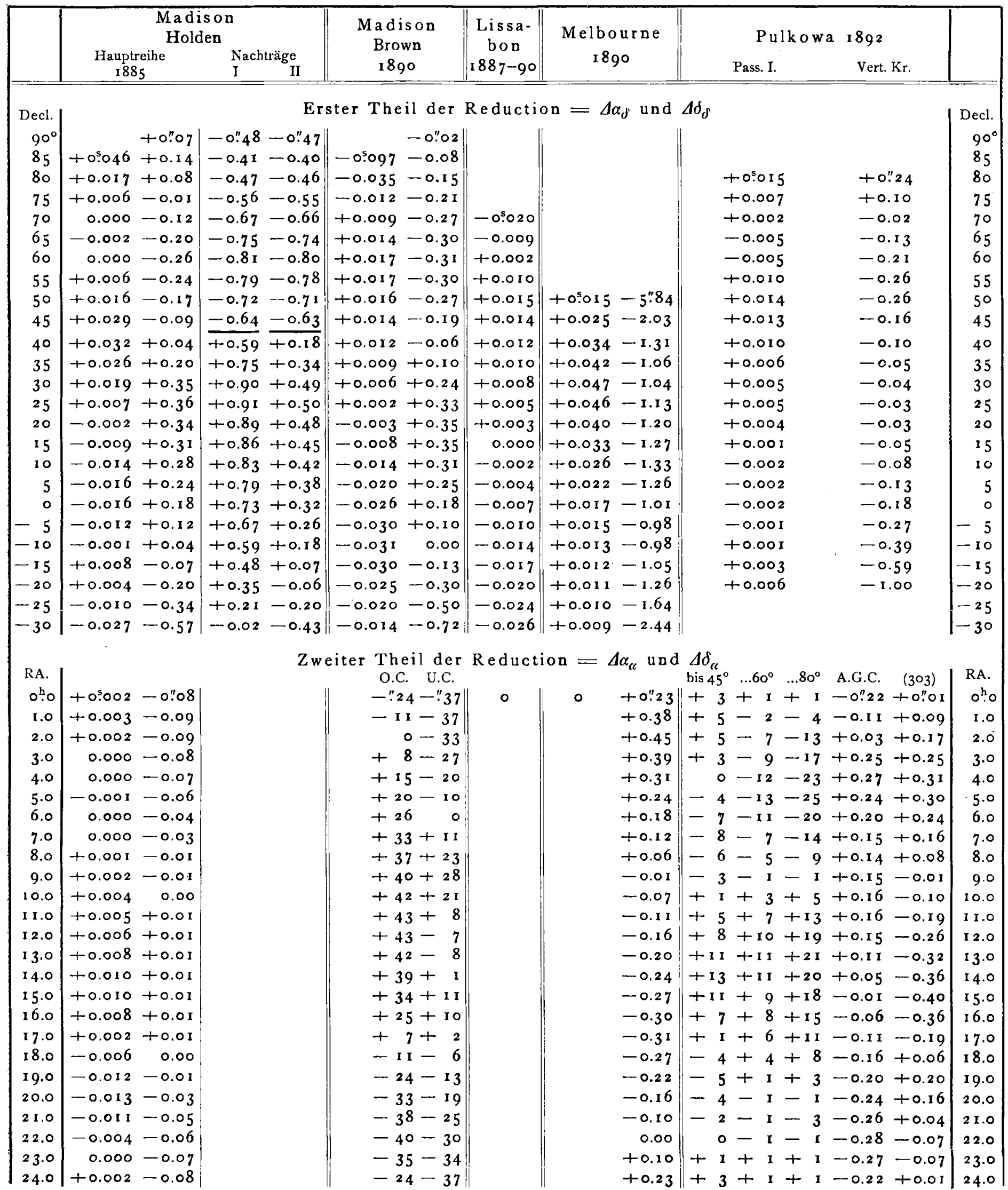




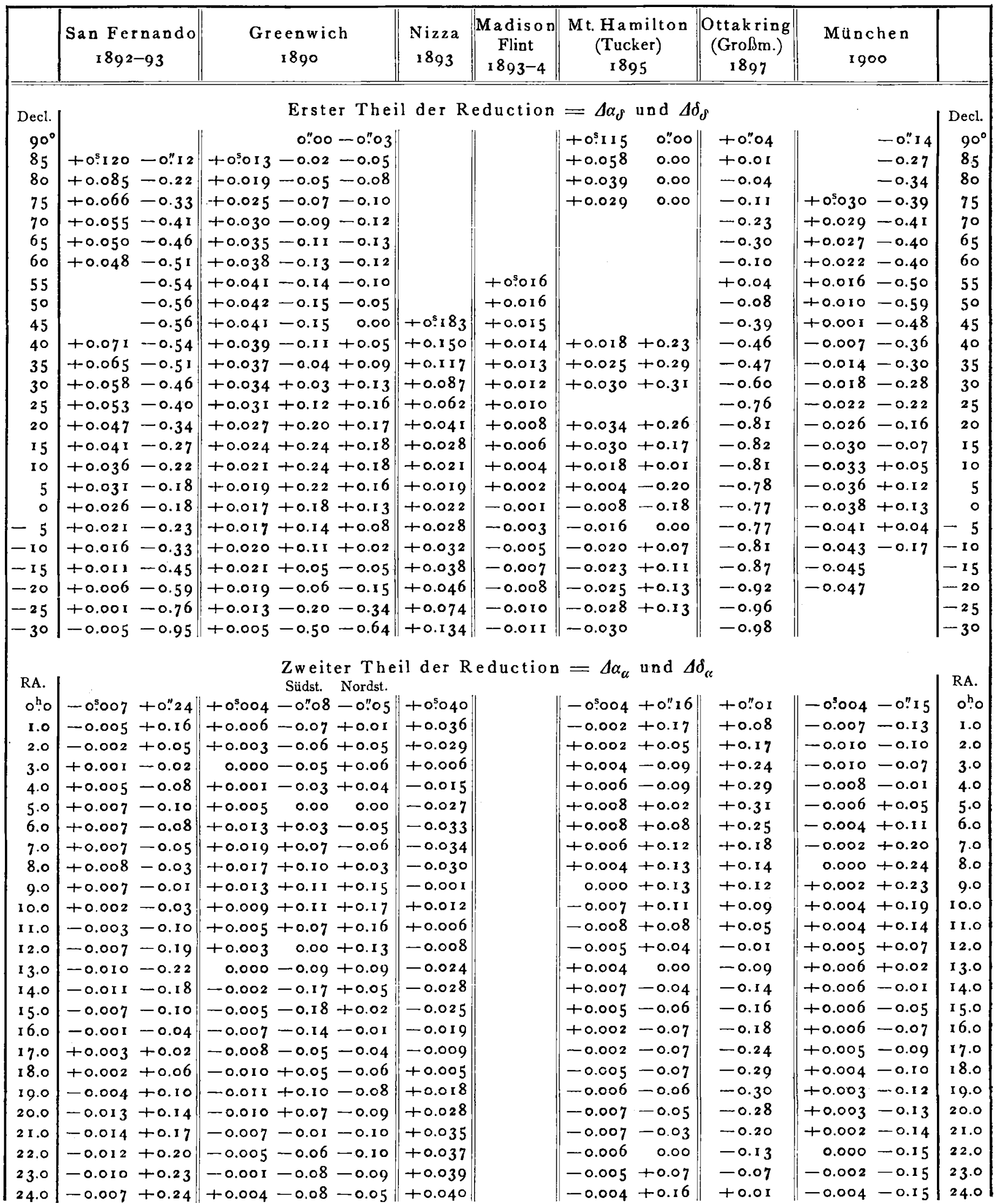


Für die Mehrzahl der Cataloge, zu denen die vor- voraussetzt. Um die hier neben einander stehenden Reihen stehenden Reductionstafeln gehören, habe ich Gewichtstafeln der $p_{\alpha}$ und $p_{\delta}$ formell gleichartig zu machen, wïrde man bereits in der Zusammenstellung A.N. 3615-6 mitgetheilt. Für also die $p_{c c}$ noch durch 2.08 zu dividiren, oder die $p_{\delta}$ mit die übrigen Cataloge kommen die folgenden hinzu, deren $2.08 \mathrm{zu}$ multipliciren haben.

Gewichtseinheit wieder die m.F. \pm 0.038 I $\sec \delta$ und \pm 0.396

\begin{tabular}{|c|c|c|c|c|c|c|c|c|c|c|c|c|c|c|c|c|c|}
\hline \multirow[b]{2}{*}{$\mid \begin{array}{c}\dot{0} \\
\vdots \\
\infty \\
\infty\end{array}$} & I. & 2. & \multicolumn{2}{|c|}{3.} & \multicolumn{2}{|c|}{4.} & \multicolumn{2}{|c|}{5.} & \multicolumn{2}{|c|}{6.} & 7 . & 8. & 9. & II. & II. & I 2. & \\
\hline & $\begin{array}{l}\text { Groombr. } \\
\text { (Thack.) }\end{array}$ & $\begin{array}{l}\text { Kön. } \\
18 \text { I5 }\end{array}$ & & $\begin{array}{l}\text { nd } \\
\text { is }\end{array}$ & & $\begin{array}{l}\text { ylor } \\
\text { wn.) }\end{array}$ & $\begin{array}{r}\text { Edir } \\
18\end{array}$ & $\begin{array}{l}\text { burg } \\
40\end{array}$ & $\begin{array}{r}\mathrm{Ma} \\
\text { G.C. }\end{array}$ & $\begin{array}{l}\text { dras } \\
\text { I } 875\end{array}$ & $\begin{array}{c}\text { Romberg } \\
1885\end{array}$ & $\begin{array}{l}\text { Liss. } \\
1889\end{array}$ & $\begin{array}{c}\text { Melbourne } \\
\text { 1 } 890\end{array}$ & $\begin{array}{l}\text { Ottakr. } \\
1897\end{array}$ & $\begin{array}{c}\text { Küstner } \\
\text { Bonn }\end{array}$ & $\begin{array}{c}\text { München } \\
\text { r } 900\end{array}$ & 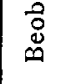 \\
\hline & RA. Decl. & Decl. & RA. & Decl & RA. & Decl. & RA. & Decl. & RA. & Decl. & RA. Decl. & RA. & RA. Decl. & Decl. & RA. Decl. & RA. Decl. & \\
\hline 1 & 0.080 .2 & 0.02 & & 0.1 & & 0.04 & 0.1 & 0.1 & 0.0 & 40.04 & $\begin{array}{lll}0.3 & 0.2 & 0.4\end{array}$ & 0.5 & 0.40 .2 & 0.2 & 1.31 .0 & 0.60 .5 & I \\
\hline 2 & $\begin{array}{ll}0.2 & 0.3\end{array}$ & 0.03 & & 0.2 & 0.1 & 0.08 & 0.2 & 0.3 & 0.06 & 90.07 & $0.7 \quad 0.3 \quad 0.7$ & 1.0 & $0.8 \quad 0.4$ & & $\begin{array}{ll}2.4 & 1.7\end{array}$ & 1.21 .0 & 2 \\
\hline 3 & $\begin{array}{ll}0.2 & 0.4\end{array}$ & 0.04 & 0.3 & 0.3 & 0.2 & 0.1 & 0.3 & 0.4 & & 0.1 & $1.00 .4 \cdot 1.0$ & I. 5 & $\begin{array}{ll}1.1 & 0.6\end{array}$ & & $3.4 \quad 2.3$ & $\begin{array}{ll}1.8 & 1.3\end{array}$ & 3 \\
\hline 4 & $\begin{array}{ll}0.2 & 0.4\end{array}$ & 0.05 & 0.4 & 0.4 & 0.2 & 0.1 & 0.4 & 0.5 & 0.2 & 0.1 & $\begin{array}{lll}1.2 & 0.5 & 1.3\end{array}$ & 2.0 & $\begin{array}{ll}1.5 & 0.8\end{array}$ & & $\begin{array}{ll}4.3 & 2.7\end{array}$ & 2.31 .5 & 4 \\
\hline 5 & 0.30 .5 & 0.06 & 0.5 & 0.5 & 0.3 & 0.2 & 0.5 & 0.6 & 0.2 & 0.2 & $\begin{array}{lll}1.5 & 0.7 & 1.5\end{array}$ & 2.4 & $\begin{array}{ll}1.8 & 0.9\end{array}$ & & 5.13 .1 & $\begin{array}{ll}2.8 & 1.7\end{array}$ & 5 \\
\hline 6 & $0.3 \quad 0.5$ & 0.06 & 0.6 & 0.6 & 0.3 & 0.2 & 0.6 & 0.7 & 0.3 & 0.2 & \begin{tabular}{|lll}
1.8 & 0.8 & 1.7
\end{tabular} & 2.8 & $2.1 \quad 1.1$ & & $5.9 \quad 3.4$ & $\begin{array}{ll}3.3 & 1.9\end{array}$ & 6 \\
\hline 7 & $\begin{array}{ll}0.4 & 0.5\end{array}$ & 0.06 & 0.7 & 0.6 & 0.4 & 0.2 & 0.7 & 0.8 & 0.3 & 0.2 & $\begin{array}{llll}2.0 & 0.9 & 1.9\end{array}$ & 3.2 & $2.4 \quad 1.3$ & I. 4 & $\begin{array}{ll}6.5 & 3.7\end{array}$ & $\begin{array}{ll}3.8 & 2.1\end{array}$ & 7 \\
\hline 8 & $0.4 \quad 0.5$ & 0.07 & 0.8 & 0.7 & 0.4 & 0.2 & 0.8 & 0.9 & 0.3 & 0.2 & 2.21 .02 .1 & 3.6 & $2.7 \quad 1.4$ & I. 5 & $\begin{array}{ll}7.1 & 3.9\end{array}$ & 4.22 .2 & 8 \\
\hline 9 & 0.40 .6 & 0.07 & 0.9 & 0.8 & 0.5 & 0.2 & 0.9 & 0.9 & 0.4 & 0.3 & $2.4 \mathrm{I} . \mathrm{I} 2.2$ & 3.9 & $2.9 \quad 1.5$ & 1.7 & $7.7 \quad 4.1$ & $\begin{array}{lll}4.7 & 2.3\end{array}$ & 9 \\
\hline 10 & $0.4 \quad 0.6$ & 0.07 & 1.0 & 0.8 & 0.5 & 0.3 & 1.0 & 1.0 & 0.4 & 0.3 & \begin{tabular}{|lll}
2.6 & $\mathrm{I} .2$ & 2.4
\end{tabular} & 4.2 & $\begin{array}{ll}3.2 & 1.7\end{array}$ & 1.8 & $\begin{array}{ll}8.2 & 4.3\end{array}$ & 5.12 .4 & 10 \\
\hline 11 & $0.4 \quad 0.6$ & 0.07 & 1.0 & 0.9 & 0.5 & 0.3 & I. I & I. I & 0.5 & 0.3 & 2.81 .22 .5 & 4.4 & $3.4 \quad 1.8$ & 2.0 & $\begin{array}{ll}8.7 & 4.4\end{array}$ & $5.5 \quad 2.5$ & I 1 \\
\hline I 2 & $0.4 \quad 0.6$ & 0.08 & I.I & 0.9 & 0.6 & 0.3 & I.1 & I. 2 & 0.5 & 0.3 & 3.01 .32 .7 & 4.9 & $3.7 \quad 2.0$ & 2.1 & $9.1 \quad 4.6$ & 5.92 .6 & $\mathrm{I}^{2}$ \\
\hline 13 & $0.5 \quad 0.6$ & 0.08 & 1.2 & 0.9 & 0.6 & 0.3 & I. 2 & 1.2 & 0.6 & 0.3 & $\begin{array}{lll}3.2 & 1.4 & 2.8\end{array}$ & 5.2 & 3.92 .1 & 2.2 & $\begin{array}{ll}9.5 & 4.7\end{array}$ & 6.32 .6 & I 3 \\
\hline$I_{4}$ & $0.5 \quad 0.6$ & 0.08 & I. 3 & 1.0 & 0.6 & 0.3 & 1.3 & T. 3 & 0.6 & 0.3 & $3.3 \times .52 .9$ & $5 \cdot 5$ & $4.1 \quad 2.2$ & 2.4 & $\begin{array}{ll}9.9 & 4.8\end{array}$ & $\begin{array}{lll}6.7 & 2.7\end{array}$ & I 4 \\
\hline 15 & 0.50 .7 & 0.08 & 1.3 & 1.0 & 0.6 & 0.3 & 1.4 & I. 4 & 0.6 & 0.4 & $3.5 \times .53 .0$ & 5. & $\begin{array}{ll}4.3 & 2.3\end{array}$ & 2.5 & 10.24 .9 & 7.02 .8 & I 5 \\
\hline I 6 & $\begin{array}{ll}0.5 & 0.7\end{array}$ & 0.08 & 1.4 & I.I & 0.7 & 0.3 & I.4 & I. 4 & 0.7 & 0.4 & 3.61 .63 .1 & 6.1 & $\begin{array}{ll}4.5 & 2.5\end{array}$ & 2.6 & $10.6 \quad 5.0$ & $7.3 \quad 2.8$ & 16 \\
\hline I 7 & $\begin{array}{ll}0.5 & 0.7\end{array}$ & 0.08 & 1.4 & I.I & 0.7 & 0.3 & I. 5 & 1.5 & 0.7 & 0.4 & $\begin{array}{llll}3.8 & 1.7 & 3.2\end{array}$ & 6.3 & $4.7 \quad 2.6$ & 2.7 & $10.9 \quad 5.0$ & $7.7 \quad 2.9$ & I 7 \\
\hline 18 & $\begin{array}{ll}0.5 & 0.7\end{array}$ & 0.08 & 1.5 & 1.1 & 0.7 & 0.4 & I.5 & I. 5 & 0.7 & 0.4 & $3.91 .7 \quad 3.3$ & 6.6 & $\begin{array}{ll}4.9 & 2.7\end{array}$ & 2.8 & 11.25 .1 & $\begin{array}{lll}8.0 & 2.9\end{array}$ & 18 \\
\hline 19 & $\begin{array}{ll}0.5 & 0.7\end{array}$ & 0.08 & 1.5 & I.2 & 0.7 & 0.4 & 1.6 & 1.6 & 0.8 & 0.4 & $4.0 \quad 1.8 \quad 3.3$ & 6.8 & $5.1 \quad 2.8$ & 2.9 & II.5 5.2 & $\begin{array}{ll}8.3 & 2.9\end{array}$ & 19 \\
\hline 20 & $\begin{array}{ll}0.5 & 0.7\end{array}$ & 0.08 & 1.6 & I. 2 & 0.8 & 0.4 & 1.7 & I.6 & 0.8 & 0.4 & $4.2 \quad 1.83 .4$ & 7.0 & $5.3 \quad 2.9$ & 3.0 & $11.7 \quad 5.3$ & 8.63 .0 & 20 \\
\hline 25 & $\begin{array}{ll}0.6 & 0.7\end{array}$ & 0.09 & 1.8 & 1.3 & 0.8 & 0.4 & I.9 & 1.8 & 1.0 & 0.5 & 4.72 .13 .7 & 8. $\mathrm{I}$ & 6.1 & 3.4 & $12.8 \quad 5.6$ & 10.03 .1 & 25 \\
\hline 30 & $0.6 \quad 0.7$ & 0.09 & 2.0 & I. 4 & 0.9 & 0.4 & 2. I & 2.0 & 1.2 & 0.5 & $\begin{array}{llll}5.2 & 2.3 & 4.0\end{array}$ & 9.0 & 6.8 & 3.8 & $\begin{array}{ll}13.6 & 5.7\end{array}$ & I I 1.23 .2 & 30 \\
\hline 40 & $\begin{array}{ll}0.6 & 0.7\end{array}$ & 0.09 & 2.3 & ז.6 & 1.0 & 0.5 & 2.5 & 2.3 & I. 5 & 0.6 & $\begin{array}{lll}5.9 & 2.6 & 4.3\end{array}$ & 10.5 & 7. & 4.4 & $14.8 \quad 6.0$ & 13.23 .4 & $4 \circ$ \\
\hline 50 & $0.6 \quad 0.8$ & 0.09 & 2.6 & 1.7 & 1.1 & 0.5 & 2.8 & 2.5 & 1.8 & 0.6 & $6.42 .8 \quad 4.6$ & 11.6 & 8.8 & 4.9 & & $14.7 \quad 3.5$ & $5^{\circ}$ \\
\hline 60 & $\begin{array}{ll}0.7 & 0.8\end{array}$ & 0.1 & 2.8 & 1.7 & I. 2 & 0.5 & 3.0 & 2.7 & 2.1 & 0.7 & $\begin{array}{lll}6.8 & 3.0 & 4.8\end{array}$ & I 2.5 & $9 \cdot 5$ & 5.2 & & & 60 \\
\hline 80 & $\begin{array}{ll}0.7 & 0.8\end{array}$ & $0 . \mathrm{I}$ & $3 \cdot 1$ & 1.8 & I. 2 & 0.5 & 3.4 & 2.9 & 2.6 & 0.7 & 7.43 .35 .0 & 13.9 & 10.5 & 5.7 & & & 80 \\
\hline 100 & $\begin{array}{ll}0.7 & 0.8\end{array}$ & & $3 \cdot 3$ & 1.9 & I. 3 & 0.6 & 3.6 & 3.1 & 3.1 & 0.7 & & 14.8 & II 1.2 & & & & 100 \\
\hline 120 & & & 3.4 & 2.0 & 1.3 & 0.6 & 3.8 & & 3.6 & 0.8 & 8.1 & & I 1.8 & & & & 20 \\
\hline 150 & & & 3.6 & 2.0 & I. 4 & 0.6 & 4.0 & & 4.0 & 0.8 & & & & & & & 50 \\
\hline 20 & & & 3.8 & 2.1 & 1.4 & 0.6 & 4.2 & & 4.7 & 0.8 & & & & & & & Do \\
\hline 300 & & & 4.0 & 2.1 & & & $4 \cdot 5$ & & & & & & & & & & ০০ \\
\hline
\end{tabular}

1. Groombridge's Catalog enthält $25^{8}$ Sterne des A.G.C. Unter den Beobachtungen bis Ende 1810 kommen davon 22 noch nicht vor.

2. I 86 gemeinschaftliche Sterne. 3. Tafel für RA. bis $-10^{\circ}$ gültig; zwischen $-10^{\circ}$ und $-20^{\circ}$ sind die Gewichte noch mit $1 / 2$, fiur südlichere Sterne mit $1 / 4$ zu multipliciren. - Tafel für Decl. giultig bis - $10^{\circ}$ oder - I $5^{\circ}$. Z Zahl der gemeinschaftlichen Sterne 597.

4. 596 gemeinschaftiche Sterne. 5. Zur Entnahme des Gewichts der Rectascensionen hat man die Anzahl der $1836-1839$ angestellten Beobachtungen mit $1 / 2$ zu multipliciren. Die Tafel fïr Decl. gilt bis $-10^{\circ}$, für südlichere Sterne sind die Gewichte herabzusetzen, für häufig beobachtete auf die Hälfte, für wenig (bis Io mal) beobachtete auf ein Viertel. - Zahl der gemeinschaftlichen Sterne 556.

6. 509 gemeinschaftliche Sterne. 7. Die erste Columne der Tafel $p_{a}$ gilt bis $-10^{\circ}$, die zweite für südlichere Sterne. Tafel $\not \partial \delta$ gilt bis $-10^{\circ}$, zwischen $-10^{\circ}$ und $-20^{\circ}$ Reductionsfactor $2 / 3$, suidlicher etwa $1 / 2$. Z Zahl der gemeinschaftlichen Sterne 600.

8. 359 Sterne des A.G.C. 9. Tafel für Decl. gültig bis $+30^{\circ}$, 2wischen $30^{\circ}$ und $40^{\circ}$ kommt der Factor $2 / 8$, nördlicher bis $Z$ D. $80^{\circ}$ $\left(+42^{\circ} \mathrm{I}\right)$ der Factor $1 / 2$ hinzu. - Zahl der gemeinschaftlichen Sterne 320.

ro. I55 Sterne des A.G.C. 1 I. 400 Sterne des A.G.C. 12. RA. von 208, Decl. von 226 Sternen des A.G.C.

Berlin I 903 Mai I.

A. Auwers. 